\title{
Engineering of Nanoscale Contrast Agents for Optical Coherence Tomography
}

Andrew Y Gordon ${ }^{1}$ and Ashwath Jayagopal ${ }^{1,2 *}$

${ }^{1}$ Department of Molecular Physiology and Biophysics, Vanderbilt University Medical Center, USA

${ }^{2}$ Vanderbilt Eye Institute, Vanderbilt University Medical Center, USA

\begin{abstract}
Optical coherence tomography has emerged as valuable imaging modalityin ophthalmology and other fields by enabling high-resolution three-dimensional imaging of tissue. In this paper, we review recent progress in the field of contrast-enhanced optical coherence tomography (OCT). We discuss exogenous and endogenous sources of OCT contrast, focusing on their use with standard OCT systems as well as emerging OCT-based imaging modalities. We include advances in the processing of OCT data that generate improved tissue contrast, including spectroscopic OCT (SOCT), as well as work utilizing secondary light sources and/or detection mechanisms to create and detect enhanced contrast, including photothermal OCT (PTOCT) and photoacoustic OCT (PAOCT). Finally, we conclude with a discussion of the translational potential of these developments as well as barriers to their clinical use.
\end{abstract}

\section{Introduction}

Since its development in the early 90s [1], optical coherence tomography (OCT) has emerged as an important imaging modality for clinicians and researchers. OCT is clinically applied primarily in the field of ophthalmology, where it offers unparalleled resolution of retinal structure and valuable information about eye disease in vivo, though its scope of application will likely expand in the future [2]. For preclinical and emerging clinical applications, it has been used to imagethe lumen of large vessels to characterize plaque formation and arterial disease, skin lesions to determine the extent of cancerous invasion and lymph nodes for detection and staging of cancer metastasis [3-7].

While all OCT systems ultimately rely on the interference of coherent light reflected from sample tissues to generate their image, usually with axial resolutions of 2-10 microns, the particular set-up and processing algorithms may vary significantly. Most OCT systems can also image blood flow in vivo utilizing its Doppler shift, the change in frequency of a wave detected by a stationary observer when it is emittedfrom a moving source. In the case of OCT, since blood is moving through the tissue being observed, it creates a detectable shift in the frequency of the light received by the OCT system that is interpreted as blood flow $[8,9]$. The most common OCT systems used in research are the newer spectral-domain OCT (SD-OCT), which allow for much faster image acquisition at improved resolutions relative toprevious generation time-domain (TD-OCT) systems. The development of ultrahigh-resolution OCT systems promises to improve image quality even further, allowing for a potential axial resolution of 1 micron [10]. This improved resolution combined with a low image acquisition time will further cement the role of OCT as a valuable in vivo imaging modality.

\section{Design of Contrast Agents for OCT}

As in Computed Tomography (CT) and Magnetic Resonance Imaging (MRI), contrast agents have been designed to allow OCT systems to image disease biomarkers and further elucidate vascular and tissue morphology for enhanced diagnostic capabilities [7,11]. However, despite OCT's widespread use and broad applicability to clinically important diseases, there are no OCT contrast agents approved for use in human patients, and thus there are no clinical approaches to image disease biomarkers using this technology, although tissue and vascular architecture can be imaged very well with OCT without the need for exogenously-administered contrast agents. OCT systems generateimages by contrasting the reflectivity of tissues with their surroundings. Unfortunately, this technique cannot readily distinguish cells with similar reflectivity in close proximity to one another, creating the need for cell-marker-specific contrast agents. Researchers generally pursue this goal by attaching "homing beacons," including antibodies or small peptides that allow for concentration of contrast agents on the surface of relevant cells [12]. In this fashion, researchers aim not only to improve contrast in OCT images of healthy tissues, but also to allow the system to image disease states based on the expression of endogenous tissue biomarkers. Such an agent would be useful for both researchers and clinicians because, combined with the high resolution of OCT imaging, contrast could offer imaging of disease states with unparalleled cell-level clarity in vivo.

\section{Design Principles of OCT Contrast Agents}

To address this need, targeted and passive (e.g. blood pooling agent) OCT contrast agents are currently being developed. The most commonly studied OCT contrast agents are gold nanoparticles, particularly gold nanorods (GNRs). GNR contrast is generated by tuning their surface plasmon resonance (SPR) frequency to the same wavelength of light emitted and interpreted by OCT systems [13]. Specifically, the SPR of a gold nanoparticle causes it to interact with light at that frequency, principally by absorbing it, although there are scattering effects as well. Combined with surface-immobilized targeting ligands to localize the particles to diseased cells of interest, these particles can be designed to offer molecularly targeted contrast for OCT systems. Generally, targeting ligands take the form of antibodies, receptors, or peptidesattached to the surface of gold nanoparticles [14-16]. Nanoparticle-ligand bonds often involve an intermediate such as polyethylene glycol, with the general goal being

*Corresponding author: Ashwath Jayagopal, Ph.D, Department of Molecular Physiology and Biophysics, Vanderbilt University Medical Center- 8010, Nashville, TN 37232, USA, Tel: 615-343-7851, E-mail: Ash.jayagopal@vanderbilt.edu

Received December 30, 2013; Accepted January 28, 2014; Published January 30, 2014

Citation: Gordon AV, Jayagopal A (2014) Engineering of Nanoscale Contrast Agents for Optical Coherence Tomography. J Nanomed Nanotechol S5:004. doi:10.4172/2157-7439.S5-004

Copyright: () 2014 Lopez Gordon AV, et al. This is an open-access article distributed under the terms of the Creative Commons Attribution License, which permits unrestricted use, distribution, and reproduction in any medium, provided the original author and source are credited. 
maintenance of nanoparticle biocompatibility while also concentrating the nanoparticle in tissue with a specific surface marker [15]. This approach has been successfully used to target nanoparticles tomultiple tissue types [16,17]. While gold is considered highly biocompatible, there have been concerns raised regarding the safety profile of GNRs, as early experimentation with their use in vivo generated considerable immune responses [12]. More recent work, however, has shown that GNR toxicity can be largely nullified by altering the composition of the molecules on their surface. In particular, cetyltrimethylammonium bromide (CTAB), a surface coating left on GNRs after their production, must be removed for the nanorods to avoid generating a strong immune response $[18,19]$. Other gold nanoparticles are being explored for their unique optical properties, notably nanocages and stellate gold nanostructures, though these particles present a greater challenge when attempting to modify their native absorption frequencies [20]. Other designs for OCT contrast agents being researched at an early stage include polystyrene (i.e. latex) microspheres, carbon nanotubes, dyes, and iron oxides [21-24].

Because most of the contrast agents mentioned previously interact with light at the frequencies used in OCT systems, they can, when appropriately targeted, act as contrast agents without further modification to the particles or the method of interpreting the data acquired by the OCT system. However, several new techniques are being studied to further improve detection of the signal generated by these contrast agents. The simplest of these to implement is spectroscopic OCT (SOCT), which analyzes the data generated by OCT systems for any shifts in light reflectivity across the range of wavelengths interpreted by an OCT system. These shifts can generate a type of natural s unevenly, they can theoretically be detected as exogenous contrast agents in this imagipectroscopic contrast in tissues [25,26]. Because GNRs affect the absorption of light across these wavelengths ng modality. The major drawback of SOCT imaging is that it requires a tissue-specific analysis of raw OCT data that is not supported by most commercially available imaging systems. However, once contrast agents for a given tissue, for example the retina, have been designed and tested with SOCT, the software used to analyze the data can be easily distributed, and likely included in commercial OCT systems in the future.

Several "active contrast" techniques are being studied for use with
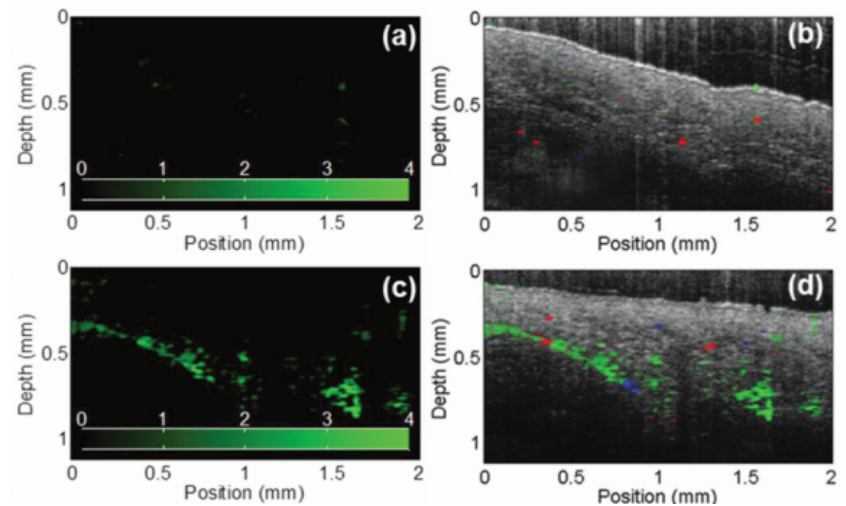

Figure 1: This figure shows images captured using a РTOCT system, demonstrating the utility of GNRs as exogenous contrast agents in a mouse ear in vivo. (a) The PTOCT signal (shown in green) generated by Matrige injected into a mouse ear as a control, (b) is overlaid on an OCT image of the same tissue, with red and blue coloration representing Doppler signals. (c) The PTOCT signal generated by 400pM GNR in Matrigel after injection into a mouse ear (d) overlaid on an OCT image of the same. Reproduced with permission from Tucker-Schwartz et al. [22].
OCT systems. These require an additional light source, but offer the potential advantage of an increased signal-to-background ratio of the relevant contrast agents in tissue. These techniques are photoacoustic OCT (PAOCT) [27], photothermal OCT (PTOCT) [28,29], and pump-probe OCT (PPOCT) [16]. Photoacoustic OCT relies on the photoacoustic effect, the emission of sound waves by certain particles after they have absorbed energy at a certain wavelength. This emitted sound can be measured by additional instrumentation and allows for localization of contrast agents overlaid on traditional OCT images [27]. Similarly, photothermal OCT relies on the ability of some particles to heat their surroundings when they absorb light at certain wavelengths. This localized tissue heating causes a shift in the index of refraction of the surrounding tissue that can be detected due to its effect on the path length of the light detected by an OCT machine [30]. Both of these techniques require a second light source in addition to the OCT source to generate the desired effect in the contrast agent. PAOCT also requires a way to measure the generated acoustic signal, while the thermal effect in PTOCT can be extracted from data collected by OCT systems without additional measurement instrumentation, though it does require analysis that isn't natively supported by commercial systems, similar to SOCT. PTOCT has been used to show contrast in mammalian tissue in vivo using GNRs, while PAOCT relies on endogenous contrast from hemoglobin in the vasculature [27-29]. The pump-probe method detects dyes, notably indocyanine green (ICG) by acquiring an OCT image, photobleaching the dye with a second light source, and reacquiring the image [23]. These approaches all have major problems with clinical applicability. In all three systems, an additional light source is required, and these sources are not standard on most OCT systems, limiting the likelihood of these methods of imaging contrast being widely adopted. Additionally, the requirement of a second measuring instrument with PAOCT currently prohibits in vivo imaging of many tissues, although it has been used for study of skin lesions (Figure 1).

One newer method of imaging contrast agents with OCT systems, magneto-motive OCT (MMOCT), uses the magnetic properties of functionalized iron oxide probes to detect the probes' motion in an externally applied magnetic field [24,31]. This has shown some utility in looking at certain tumor models, but is hindered in its clinical applicability by its requiring an external magnetic field.

\section{Outlook}

The future for OCT contrast agents in clinical applications is promising. Since researchers are already able to image GNR contrast agents natively using commercially available OCT systems, they will continue to be prominent in translational research in this field and will likely constitute the first line of clinically available OCT contrast agents, provided that their toxicity concerns are alleviated. Critical barriers to translation will involve demonstrations in patients that contrast agentguided OCT is capable of earlier detection of diseases compared to conventional imaging methods, as well as achievement of high signal to noise ratios to minimize false positives in diagnostic imaging.

\section{References}

1. Huang D, Swanson EA, Lin CP, Schuman JS, Stinson WG, et al. (1991) Optical coherence tomography. Science 254: 1178-1181.

2. Wali U, Kharousi N (2012) Clinical applications of optical coherence tomography in ophthalmology. Intech 11: 197-238.

3. Liu L, Gardecki JA, Nadkarni SK, Toussaint JD, Yagi Y, et al. (2011) Imaging the subcellular structure of human coronary atherosclerosis using micro-optical coherence tomography. Nat Med 17: 1010-1014.

4. Farooq MU, Khasnis A, Majid A, Kassab MY (2009) The role of optical coherence tomography in vascular medicine. Vasc Med 14: 63-71. 
Citation: Gordon AV, Jayagopal A (2014) Engineering of Nanoscale Contrast Agents for Optical Coherence Tomography. J Nanomed Nanotechol S5:004. doi:10.4172/2157-7439.S5-004

5. Alex A, Povazay B, Hofer B, Popov S, Glittenberg C et al. (2010) Multispectral in vivo three-dimensional optical coherence tomography of human skin. $J$ Biomed Opt 15: 026025.

6. Chan CS, Rohrer TE (2012) Optical coherence tomography and its role in mohs micrographic surgery: a case report. Case Rep Dermatol 4: 269-274.

7. Jung Y, Reif R, Zeng Y, Wang RK (2011) Three-dimensional high-resolution imaging of gold nanorods uptake in sentinel lymph nodes. Nano Lett 11: 29382943

8. Liu G, Chen Z (2013) Advances in Doppler OCT. Chin Opt Lett 11: 11702.

9. Liu G, Jia W, Nelson JS, Chen Z (2013) In vivo, high-resolution, threedimensional imaging of port wine stain microvasculature in human skin. Lasers Surg Med 45: 628-632

10. Drexler W, Morgner U, Ghanta RK, Kärtner FX, Schuman JS, et al. (2001) Ultrahigh-resolution ophthalmic optical coherence tomography. Nat Med 7 : 502-507.

11. Skala MC, Crow MJ, Wax A, Izatt JA (2008) Photothermal optical coherence tomography of epidermal growth factor receptor in live cells using immunotargeted gold nanospheres. Nano Lett 8: 3461-3467.

12. Gabriele Sandrian M, Wollstein G, Schuman JS, Bilonick RA, Ling Y, et al (2012) Inflammatory response to intravitreal injection of gold nanorods. $\mathrm{Br} J$ Ophthalmol 96: 1522-1529.

13. Troutman TS, Barton JK, Romanowski M (2007) Optical coherence tomography with plasmon resonant nanorods of gold. Opt Lett 32: 1438-1440.

14. Wang J, Dong B, Chen B, Jiang Z, Song H (2012) Selective photothermal therapy for breast cancer with targeting peptide modified gold nanorods. Dalton Trans 41: 11134-11144.

15. Choi J, Park Y, Choi EB, Kim HO, Kim DJ, et al. (2014) Aptamer-conjugated gold nanorod for photothermal ablation of epidermal growth factor receptoroverexpressed epithelial cancer. J Biomed Opt 19: 051203.

16. Schol D, Fleron M, Greisch JF, Jaeger M, Frenz M, et al. (2013) Anti-PSMA antibody-coupled gold nanorods detection by optical and electron microscopies. Micron 50: 68-74.

17. Liopo A, Conjusteau A, Tsyboulski D, Ermolinsky B, Kazansky A, et al. (2012) Biocompatible Gold Nanorod Conjugates for Preclinical Biomedical Research. J Nanomed Nanotechnol S2.

18. Leonov AP, Zheng J, Clogston JD, Stern ST, Patri AK, et al. (2008) Detoxification of gold nanorods by treatment with polystyrenesulfonate. ACS Nano 2: 24812488.
19. Lee SE, Sasaki DY, Perroud TD, Yoo D, Patel KD, et al. (2009) Biologically functional cationic phospholipid-gold nanoplasmonic carriers of RNA. J Am Chem Soc 131: 14066-14074.

20. Hu M, Chen J, Li ZY, Au L, Hartland GV, et al. (2006) Gold nanostructures: engineering their plasmonic properties for biomedical applications. Chem Soc Rev 35: 1084-1094.

21. Tucker-Schwartz JM, Hong T, Colvin DC, Xu Y, Skala MC (2012) Dual-modality photothermal optical coherence tomography and magnetic-resonance imaging of carbon nanotubes. Opt Lett 37: 872-874.

22. Lee TM, Oldenburg AL, Sitafalwalla S, Marks DL, Luo W, et al. (2003) Engineered microsphere contrast agents for optical coherence tomography. Opt Lett 28: 1546-1548.

23. Yaqoob Z, McDowell E, Wu J, Heng X, Fingler J, et al. (2006) Molecular contras optical coherence tomography: A pump-probe scheme using indocyanine green as a contrast agent. J Biomed Opt 11: 054017.

24. John R, Rezaeipoor R, Adie SG, Chaney EJ, Oldenburg AL, et al. (2010) In vivo magnetomotive optical molecular imaging using targeted magnetic nanoprobes. Proc Natl Acad Sci U S A 107: 8085-8090.

25. Morgner U, Drexler W, Kärtner FX, Li XD, Pitris C, et al. (2000) Spectroscopic optical coherence tomography. Opt Lett 25: 111-113.

26. Wang C, Kim J, Jin CT, Philip JHW, McEwan A (2012) Near infrared spectroscopy in optical coherence tomography. J. Near Infrared Spectrosc. 20: 237-247.

27. Zhang EZ, Povazay B, Laufer J, Alex A, Hofer B, et al. (2011) Multimodal photoacoustic and optical coherence tomography scanner using an all optical detection scheme for 3D morphological skin imaging. Biomed Opt Express 2 : 2202-2215.

28. Adler DC, Huang SW, Huber R, Fujimoto JG (2008) Photothermal detection of gold nanoparticles using phase-sensitive optical coherence tomography. Opt Express 16: 4376-4393.

29. Tucker-Schwartz JM, Meyer TA, Patil CA, Duvall CL, Skala MC (2012) In vivo photothermal optical coherence tomography of gold nanorod contrast agents. Biomed Opt Express 3: 2881-2895

30. Kim J, Oh J, Milner TE (2006) Measurement of optical path length change following pulsed laser irradiation using differential phase optical coherence tomography. J Biomed Opt 11: 041122.

31. Oldenburg A, Gunther J, Toublan F, Mark DL, Suslith KS, et al. (2004) Magnetic contrast agents for optical coherence tomography. Proc. of SPIE 5316: 91-98.
This article was originally published in a special issue, Nanotechnology: Challenges \& Perspectives in Medicine handled by Editor(s). Dr. Malavosklish Bikram, University of Houston, USA 